\title{
Parametric Resonance of Hopf Bifurcation
}

\author{
RICHARD RAND $^{1, *}$, ALBERT BARCILON $^{2}$, and TINA MORRISON ${ }^{1}$ \\ ${ }^{1}$ Department of Theoretical \& Applied Mechanics, Cornell University, Ithaca, NY 14853, U.S.A.; ${ }^{2}$ Department of Meteorology \\ and GFDI, Florida State University, Tallahassee, FL 32306, U.S.A.; *Author for correspondence (e-mail: rhr2@cornell.edu; \\ fax: $+1-607-255-2011)$
}

(Received: 23 January 2004; accepted: 27 August 2004)

\begin{abstract}
We investigate the dynamics of a system consisting of a simple harmonic oscillator with small nonlinearity, small damping and small parametric forcing in the neighborhood of 2:1 resonance. We assume that the unforced system exhibits the birth of a stable limit cycle as the damping changes sign from positive to negative (a supercritical Hopf bifurcation). Using perturbation methods and numerical integration, we investigate the changes which occur in long-time behavior as the damping parameter is varied. We show that for large positive damping, the origin is stable, whereas for large negative damping a quasi-periodic behavior occurs. These two steady states are connected by a complicated series of bifurcations which occur as the damping is varied.
\end{abstract}

Key words: parametric excitation, resonance, Hopf bifurcation

\section{Introduction}

It is well known that a limit cycle may be born in a process called a Hopf bifurcation. A typical setting is given by the autonomous equation:

$$
\ddot{z}+z+\epsilon A \dot{z}+\epsilon\left(\beta_{1} z^{3}+\beta_{2} z^{2} \dot{z}+\beta_{3} z \dot{z}^{2}+\beta_{4} \dot{z}^{3}\right)=0
$$

where $\epsilon \ll 1$ is a perturbation parameter. Here $\epsilon A$ is a linear damping coefficient and the $\epsilon \beta_{i}$ are coefficients of nonlinear terms. Perturbation theory [1] shows that Equation (1) exhibits a limit cycle with amplitude $r$, where

$$
r^{2}=-\frac{4 A}{3 \beta_{4}+\beta_{2}}
$$

In the case that $3 \beta_{4}+\beta_{2}>0$, Equation (2) gives that the limit cycle occurs for $A<0$. From (1), the origin $z=\dot{z}=0$ is unstable for $A<0$ (negative damping), and so the limit cycle, which has the opposite stability from that of the origin, is in this case stable, a situation which is referred to as a supercritical Hopf.

In this work we shall be interested in what happens when the system (1) is parametrically excited at close to twice the natural frequency of the linearized undamped system:

$$
\ddot{z}+\left(1+\epsilon k_{1}+\epsilon B \cos 2 t\right) z+\epsilon A \dot{z}+\epsilon\left(\beta_{1} z^{3}+\beta_{2} z^{2} \dot{z}+\beta_{3} z \dot{z}^{2}+\beta_{4} \dot{z}^{3}\right)=0
$$

In Equation (3), $\epsilon k_{1}$ is a detuning coefficient and $\epsilon B$ is the amplitude of parametric forcing. 


\section{R. Rand et al.}

The linearized undamped version of Equation (3) is known as Mathieu's equation:

$$
\ddot{z}+\left(1+\epsilon k_{1}+\epsilon B \cos \omega t\right) z=0
$$

where $\omega$ is the frequency of the parametric excitation. It is well known [1] that for small $\epsilon$ the largest instability occurs for $\omega=2$, a situation referred to as parametric resonance, which motivates our choice of $\omega=2$ in Equation (3).

Our interest in this problem is motivated by two applications. The first is a model of the El Nino Southern Oscillation (ENSO) coupled tropical ocean-atmosphere weather phenomenon [2,3] in which the state variables are temperature and depth of a region of the ocean called the thermocline. The annual seasonal cycle is the parametric excitation. The model exhibits a Hopf bifurcation in the absence of parametric excitation.

The second application involves a MEMS device [4] consisting of a $30 \mu \mathrm{m}$ diameter silicon disk which can be made to vibrate by heating it with a laser beam resulting in a Hopf bifurcation. The parametric excitation is provided by making the laser beam intensity vary periodically in time.

\section{Perturbation Method}

In order to investigate the dynamics of Equation (3) for small $\epsilon$, we will use the two-variable expansion method (also known as multiple scales) [1,6]. We define two new time scales, $\xi=t$ and $\eta=\epsilon t$. Here $\eta$ is referred to as slow time. Equation (3) becomes:

$$
\begin{aligned}
z_{\xi \xi} & +2 \epsilon z_{\xi \eta}+\left(1+\epsilon k_{1}+\epsilon B \cos 2 \xi\right) z+\epsilon A z_{\xi} \\
& +\epsilon\left(\beta_{1} z^{3}+\beta_{2} z^{2} z_{\xi}+\beta_{3} z z_{\xi}^{2}+\beta_{4} z_{\xi}^{3}\right)=O\left(\epsilon^{2}\right)
\end{aligned}
$$

Next we expand $z=z_{0}+\epsilon z_{1}+O\left(\epsilon^{2}\right)$ and collect terms, giving:

$$
\begin{aligned}
z_{0 \xi \xi}+z_{0}= & 0 \\
z_{1 \xi \xi}+z_{1}= & -2 z_{0 \xi \eta}-k_{1} z_{0}-B z_{0} \cos 2 \xi-A z_{0 \xi} \\
& -\left(\beta_{1} z_{0}{ }^{3}+\beta_{2} z_{0}{ }^{2} z_{0 \xi}+\beta_{3} z_{0} z_{0 \xi}{ }^{2}+\beta_{4} z_{0 \xi}{ }^{3}\right)
\end{aligned}
$$

We take the solution of (6) to be

$$
z_{0}=u \cos \xi+v \sin \xi
$$

where $u$ and $v$ depend only on slow time $\eta$. Substituting (8) into (7) and removing secular terms gives the following slow flow:

$$
\begin{aligned}
& u_{\eta}=-\frac{A}{2} u+\left(\frac{k_{1}}{2}-\frac{B}{4}\right) v+\frac{1}{8}\left(\Gamma_{1} v-\Gamma_{2} u\right)\left(u^{2}+v^{2}\right) \\
& v_{\eta}=-\frac{A}{2} v+\left(-\frac{k_{1}}{2}-\frac{B}{4}\right) u+\frac{1}{8}\left(-\Gamma_{1} u-\Gamma_{2} v\right)\left(u^{2}+v^{2}\right)
\end{aligned}
$$


where

$$
\Gamma_{1}=3 \beta_{1}+\beta_{3}, \quad \Gamma_{2}=\beta_{2}+3 \beta_{4}
$$

In polar coordinates $u=r \cos \theta, v=r \sin \theta$ the slow flow (9) and (10) becomes:

$$
\begin{aligned}
& r_{\eta}=-\frac{r}{8}\left(4 A+\Gamma_{2} r^{2}+2 B \sin 2 \theta\right) \\
& \theta_{\eta}=-\frac{1}{8}\left(4 k_{1}+\Gamma_{1} r^{2}+2 B \cos 2 \theta\right)
\end{aligned}
$$

where $r>0$ and $\theta$ depend only on slow time $\eta$.

\section{Analysis of the Slow Flow}

From (8), the nontrivial $(r \neq 0)$ equilibria of the slow flow (12) and (13) correspond to limit cycles in the original Equation (3). These equilibria satisfy the equations:

$$
\begin{aligned}
& 4 A+\Lambda \Gamma_{1} \rho+2 B \sin 2 \theta=0 \\
& 4 \kappa A+\Gamma_{1} \rho+2 B \cos 2 \theta=0
\end{aligned}
$$

where we have set:

$$
\rho=r^{2}, \quad k_{1}=\kappa A, \quad \Lambda=\frac{\Gamma_{2}}{\Gamma_{1}}=\frac{\beta_{2}+3 \beta_{4}}{3 \beta_{1}+\beta_{3}}
$$

Note that although $\rho=r^{2}$ must be positive for real solutions, there are no sign restrictions on $\kappa$ and $\Lambda$.

Solving (14) and (15) respectively for $\sin 2 \theta$ and $\cos 2 \theta$, and using the identity $\sin ^{2} 2 \theta+\cos ^{2} 2 \theta=1$, we obtain the following condition on $\rho$ :

$$
\left(\Lambda^{2}+1\right) \Gamma_{1}^{2} \rho^{2}+8 A(\Lambda+\kappa) \Gamma_{1} \rho+4\left[4 A^{2}\left(1+\kappa^{2}\right)-B^{2}\right]=0
$$

Equation (17) is a quadratic on $\rho$. For real roots, the discriminant must be positive. This turns out to give the condition:

$$
\frac{A^{2}}{B^{2}}<\frac{1}{4} \frac{1+\Lambda^{2}}{(1-\kappa \Lambda)^{2}}
$$

From this we are led to define $P=A / B$, being the ratio of linear damping coefficient $A$ to parametric forcing amplitude B. $P$ turns out to be an important bifurcation parameter for this system.

In addition, $\rho=r^{2}$ must be nonnegative. Allowable (nonnegative) values of $\rho$ will be separated from rejected (negative) values of $\rho$ by the condition $\rho=0$, which gives, from (17):

$$
P^{2}=\frac{A^{2}}{B^{2}}=\frac{1}{4\left(1+\kappa^{2}\right)}
$$




\section{R. Rand et al.}

If we fix the parameters $\beta_{i}$ and $\kappa$, then we may envision a line parameterized by $P=A / B$ which contains 4 key bifurcation points, $P_{1} \leq P_{2}<P_{3} \leq P_{4}$ :

$$
\begin{aligned}
& P_{1}=-\frac{1}{2} \sqrt{\frac{1+\Lambda^{2}}{(1-\kappa \Lambda)^{2}}}, \quad P_{2}=-\frac{1}{2} \frac{1}{\sqrt{\left(1+\kappa^{2}\right)}}, \\
& P_{3}=\frac{1}{2} \frac{1}{\sqrt{\left(1+\kappa^{2}\right)}}, \quad P_{4}=\frac{1}{2} \sqrt{\frac{1+\Lambda^{2}}{(1-\kappa \Lambda)^{2}}}
\end{aligned}
$$

From Equations (18) and (19) we see that each of these points may represent a change in the number of slow flow equilibria. Equation (18) tells us that there are no nontrivial slow flow equilibria to the left of $P_{1}$ and to the right of $P_{4}$. It also tells us that there are two real roots for $\rho=r^{2}$ in the interval between $P_{1}$ and $P_{4}$. However, this does not mean that there are two allowable values of $r>0$ in this region because some or all of these roots $\rho$ may be negative.

We will now appeal to Descartes' Rule of Signs to draw conclusions as to the number of admissible values of $r$ in the interval between $P_{1}$ and $P_{4}$. To be specific, we will assume $(\Lambda+\kappa) \Gamma_{1}$ and $B$ are positive, although similar conclusions can be drawn in the general case. Consider first the case that $A>0$. If $P>P_{3}$ then the signs in Equation (17) are +++ which tells us that there are no positive roots $\rho$ to the right of $P_{3}$, and hence no nontrivial slow flow equilibria to the right of $P_{3}$. If $0<P<P_{3}$ then the signs in Equation (17) are ++- which tells us that there is one positive and one negative root $\rho$, which means there is a single admissible value of $r$ in the interval between $P=0$ and $P=P_{3}$.

Next let us consider the case that $A<0$. If $-P_{2}<P<0$ the signs are +-- which again means that there is a single admissible value of $r$, this time in the interval between $P=P_{2}$ and $P=0$. On the other hand, if $P<P_{2}$ the signs are +-+ which means that there are no negative roots $\rho$ in the interval between $P_{1}$ and $P_{2}$. However, we have seen that there are 2 real roots $\rho$ in this interval (from the positiveness of the discriminant), and thus we may conclude that there are two positive roots $\rho$ and thus two admissible values for $r$ between $P_{1}$ and $P_{2}$.

Now it turns out that each of the admissible values of $r$ corresponds to a pair of nontrivial slow flow equilibria. This may be seen by considering the value of $\theta$ at these equilibrium points. Eliminating $\rho$ from Equations (14) and (15) gives

$$
-\sin 2 \theta+\Lambda \cos 2 \theta=2 \frac{A}{B}(1-\Lambda \kappa)
$$

which may be written as

$$
\cos (2 \theta+\psi)=2 \frac{A}{B} \frac{1-\Lambda \kappa}{\sqrt{1+\Lambda^{2}}}
$$

where the phase angle $\psi$ satisfies $\cot \psi=\Lambda$. Now if $A / B$ in Equation (22) corresponds to a value which gives a real positive value for $r$, then Equation (22) gives two values for $\theta$ which differ by 180 degrees. Thus each admissible value of $r$ found above corresponds to two slow flow equilibria which are located symmetrically with respect to the origin.

In summary then, we have shown that the number of slow flow equilibria (including the origin $r=0$ ) depends on the value of $P=A / B$, as follows:

$\left[-\infty, P_{1}\right] 1$ equilibrium

$\left[P_{1}, P_{2}\right] 5$ equilibria 
$\left[P_{2}, P_{3}\right] 3$ equilibria

$\left[P_{3},+\infty\right] 1$ equilibrium

This chart is based on the following assumptions which we have made on parameters:

$$
(\Lambda+\kappa) \Gamma_{1}>0, \quad \Gamma_{2}=\Lambda \Gamma_{1}>0, \quad B>0, \quad \epsilon \ll 1
$$

Thus we may say that as $A / B$ is decreased, a pitchfork bifurcation occurs as $P_{3}$ is crossed, resulting in three slow flow equilibria (the origin and the pair just born). Continuing to decrease $A / B$, another pitchfork occurs as $P_{2}$ is crossed, and now there are five slow flow equilibria. Finally a pair of saddlenode bifurcations occur as $P_{1}$ is crossed, in which all of the four slow flow equilibria which were created in the two pitchforks now come together in pairs and disappear. The result is that only the origin is left.

This summarizes the occurrence of slow flow equilibria. What about limit cycles in the slow flow phase plane? One way that limit cycles can occur is through Hopf bifurcations off of slow flow equilibria. The condition for a Hopf can be stated in terms of the $2 \times 2$ matrix $M$ of the linearized slow flow about an equilibrium point. For a $\operatorname{Hopf}, \operatorname{trace}(M)=0$ and $\operatorname{det}(M)>0$ (see [1] for example). From Equations (9) and (10), we obtain the following expression for $M$ :

$$
M=\left[\begin{array}{ll}
M_{11} & M_{12} \\
M_{21} & M_{22}
\end{array}\right]
$$

where

$$
\begin{aligned}
& M_{11}=-\frac{A}{2}-\frac{3}{8} \Gamma_{2} u^{2}-\frac{1}{8} \Gamma_{2} v^{2}+\frac{1}{4} \Gamma_{1} u v \\
& M_{12}=\frac{k_{1}}{2}-\frac{B}{4}+\frac{1}{8} \Gamma_{1} u^{2}+\frac{3}{8} \Gamma_{1} v^{2}-\frac{1}{4} \Gamma_{2} u v \\
& M_{21}=-\frac{k_{1}}{2}-\frac{B}{4}-\frac{1}{8} \Gamma_{1} v^{2}-\frac{3}{8} \Gamma_{1} u^{2}-\frac{1}{4} \Gamma_{2} u v \\
& M_{22}=-\frac{A}{2}-\frac{3}{8} \Gamma_{2} v^{2}-\frac{1}{8} \Gamma_{2} u^{2}-\frac{1}{4} \Gamma_{1} u v
\end{aligned}
$$

where $u$ and $v$ are to be evaluated at the slow flow equilibrium. From (24) we obtain:

$$
\operatorname{trace}(M)=-A-\frac{1}{2} \Gamma_{2} \rho
$$

Setting the $\operatorname{trace}(M)=0$ for a Hopf, we find:

$$
\operatorname{trace}(M)=0 \Rightarrow A=-\frac{1}{2} \Gamma_{2} \rho
$$

and since $\rho \geq 0$, we note that for a Hopf, $A$ must have the opposite sign of $\Gamma_{2}$, in agreement with Equation (2).

Next we solve (25) for $\rho$ and substitute it into (17), obtaining the condition:

$$
P_{\mathrm{Hopf}}^{2}=\frac{1}{1+\frac{1}{\Lambda^{2}}+4 \kappa\left(\kappa-\frac{1}{\Lambda}\right)}
$$


Condition (27) is necessary for a Hopf bifurcation, but it is not sufficient. We also need to require that $\operatorname{det}(M)>0$. From Equation (24), we obtain:

$$
\begin{aligned}
\operatorname{det}(M)= & \frac{A^{2}}{4}-\frac{B^{2}}{16}+\frac{k_{1}^{2}}{4}+\left(\frac{A \Gamma_{2}}{4}-\frac{B \Gamma_{1}}{16}+\frac{k_{1} \Gamma_{1}}{4}\right) \rho \\
& +\frac{3}{64}\left(\Gamma_{1}^{2}+\Gamma_{2}^{2}\right) \rho^{2}+\frac{B \Gamma_{1}}{8} v^{2}-\frac{B \Gamma_{2}}{8} u v
\end{aligned}
$$

This expression may be simplified by multiplying the RHS of Equation (9) by $u$ and adding it to the RHS of (10) multiplied by $v$, which gives:

$$
u v=-\frac{\rho\left(4 A+\Gamma_{2} \rho\right)}{4 B}
$$

Similarly we multiply the RHS of (9) by $v$ and subtract from it the RHS of (10) multiplied by $u$, which gives:

$$
v^{2}=\frac{\rho\left(4 k_{1}+2 B+\Gamma_{1} \rho\right)}{4 B}
$$

Substituting (29) and (30) into (28), we obtain the following expression for $\operatorname{det}(M)$ :

$$
\operatorname{det}(M)=\frac{A^{2}}{4}-\frac{B^{2}}{16}+\frac{k_{1}^{2}}{4}+\frac{3}{8}\left(A \Gamma_{2}+k_{1} \Gamma_{1}\right) \rho+\frac{5}{64}\left(\Gamma_{1}^{2}+\Gamma_{2}^{2}\right) \rho^{2}
$$

The condition $\operatorname{det}(M)=0$ corresponds to saddle-node bifurcations. Eliminating $\rho$ between $\operatorname{det}(M)=0$ in Equation (31) and the slow flow equilibrium condition (17) gives:

$$
\left(4\left(\kappa^{2}+1\right) A^{2}-B^{2}\right)\left(4 A^{2} \kappa^{2} \Lambda^{2}-B^{2} \Lambda^{2}-8 A^{2} \kappa \Lambda-B^{2}+4 A^{2}\right)=0
$$

Solving (32) for $P^{2}=A^{2} / B^{2}$, we obtain:

$$
P^{2}=\frac{A^{2}}{B^{2}}=\frac{1}{4\left(1+\kappa^{2}\right)}, \frac{1+\Lambda^{2}}{4(1-\kappa \Lambda)^{2}}
$$

in agreement with the values computed in Equations (18) and (19).

\section{Example 1}

We take as an example the following system:

$$
\ddot{z}+(1+\epsilon \cos 2 t) z+\epsilon A \dot{z}+\epsilon\left(-2 z^{3}+\dot{z}^{3}\right)=0
$$

This corresponds to the choice of parameters $\beta_{1}=-2, \beta_{2}=0, \beta_{3}=0, \beta_{4}=1, \kappa=0, B=1$ which gives $\Gamma_{1}=-6, \Gamma_{2}=3$ and $\Lambda=-1 / 2$. From Equations (20) we obtain:

$$
P_{1}=-\frac{\sqrt{5}}{4}, \quad P_{2}=-\frac{1}{2}, \quad P_{3}=\frac{1}{2}, \quad P_{4}=\frac{\sqrt{5}}{4}
$$


and from Equation (27) we find:

$$
P_{\mathrm{Hopf}}^{2}=\frac{1}{5}
$$

This system has the single parameter $A$, and from the foregoing analysis we can say that the number of slow flow equilibria changes with $A$ as follows:

$$
\begin{gathered}
{[-\infty,-0.559] 1 \text { equilibrium }} \\
{[-0.559,-0.5] 5 \text { equilibria }} \\
{[-0.5,0.5] 3 \text { equilibria }} \\
{[0.5,+\infty] 1 \text { equilibrium }}
\end{gathered}
$$

From (36), a Hopf bifurcation may occur when $A=-1 / \sqrt{5}=-0.447$, if $\operatorname{det}(M)>0$. (Here we used the fact that $A$ must have the opposite sign to $\Gamma_{2}$ for a Hopf.) In order to compute $\operatorname{det}(M)$ from Equation (31), we need $\rho$ at the Hopf. From (25) we find that $\rho=\frac{2}{3 \sqrt{5}}$, and substituting this value for $\rho$ into (31), we find $\operatorname{det}(M)=3 / 20>0$.

In order to confirm these results, and to determine any other bifurcations which occur in (37) as $A$ is varied, we numerically integrated the slow flow Equations (9) and (10) for the present parameters (see Figure 1). We found that the limit cycles born in the Hopf at $A=-0.447$ are unstable and exist in the range $-0.447 \leq A \leq-0.377$ (region IV of Figure 1). As $A$ is increased beyond -0.377 , a symmetry-breaking bifurcation occurs in which each of the two limit cycles born in the Hopf unite into one large unstable limit cycle which is point-symmetric about the origin in the $u-v$ plane (region III of Figure 1). Further increases in $A$ increase the size of the symmetric limit cycle until $A=-0.364$, at which point it coalesces with a larger stable limit cycle in a limit cycle fold.

\section{Example 2}

The following example exhibits qualitatively different behavior from Example 1 above:

$$
\ddot{z}+(1-\epsilon A+\epsilon \cos 2 t) z+\epsilon A \dot{z}+\epsilon\left(-2 z^{3}+\dot{z}^{3}\right)=0
$$

This corresponds to the choice of parameters $\beta_{1}=-2, \beta_{2}=0, \beta_{3}=0, \beta_{4}=1, \kappa=-1, B=1$ which gives $\Gamma_{1}=-6, \Gamma_{2}=3$ and $\Lambda=-1 / 2$. From Equations (20) we obtain:

$$
P_{1}=-\frac{\sqrt{5}}{2}, \quad P_{2}=-\frac{1}{2 \sqrt{2}}, \quad P_{3}=\frac{1}{2 \sqrt{2}}, \quad P_{4}=\frac{\sqrt{5}}{2}
$$

and from Equation (27) we find:

$$
P_{\mathrm{Hopf}}^{2}=1
$$

As with Example 1, this system has the single parameter $A$, and from the foregoing analysis we can say that the number of slow flow equilibria changes with $A$ as follows:

$$
\begin{array}{r}
{[-\infty,-1.118] 1 \text { equilibrium }} \\
{[-1.118,-0.3535] 5 \text { equilibria }}
\end{array}
$$




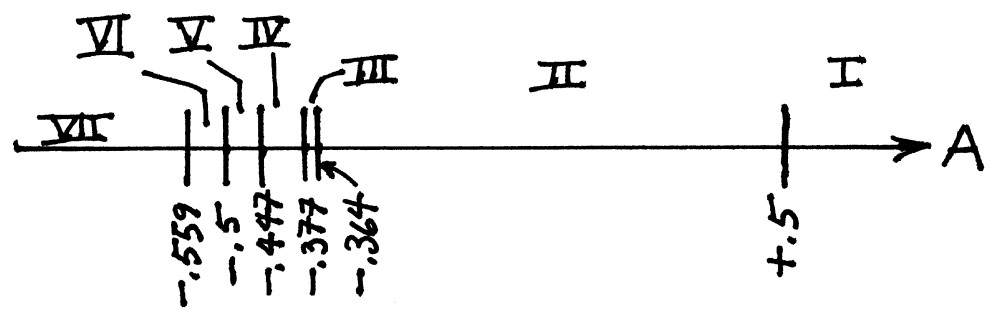

I
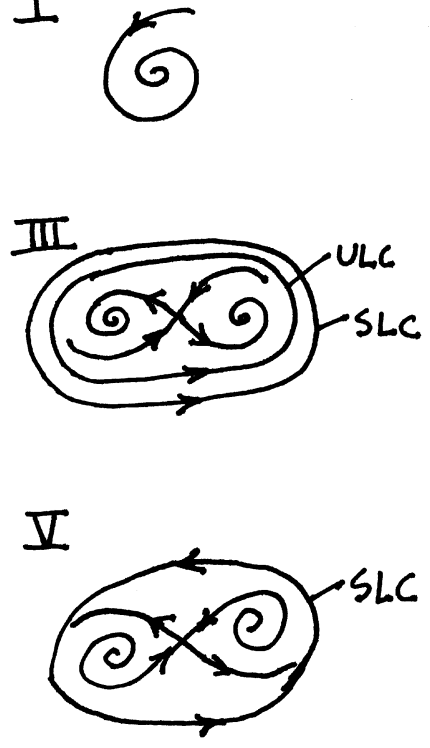

III

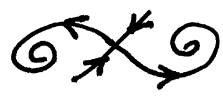

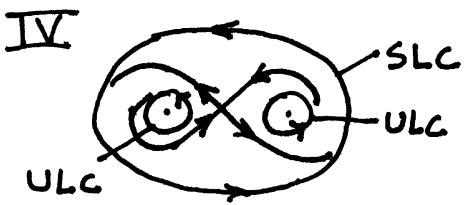

III

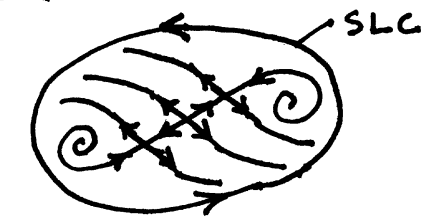

VII

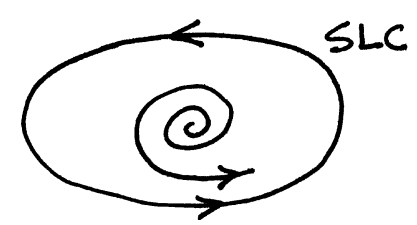

Figure 1. Sketch of slow flows in the $u-v$ plane for Example 1. ULC: unstable limit cycle, SLC: stable limit cycle.

\section{$[-0.3535,0.3535] 3$ equilibria}

$[0.3535,+\infty] 1$ equilibrium

From (39), a Hopf bifurcation may occur when $A=-1$, if $\operatorname{det}(M)>0$. (Here we again used the fact that $A$ must have the opposite sign to $\Gamma_{2}$ for a Hopf.) In order to compute $\operatorname{det}(M)$ from Equation (31), we need $\rho$ at the Hopf. From (25) we find that $\rho=\frac{2}{3}$, and substituting this value for $\rho$ into (31), we find $\operatorname{det}(M)=-1 / 4<0$. Thus there is no Hopf in this example.

Numerical integration of the slow flow Equations (9) and (10) for the present parameters reveals that a stable limit cycle is born in a saddle connection bifurcation at $A=-1.012$ (see Figure 2). 


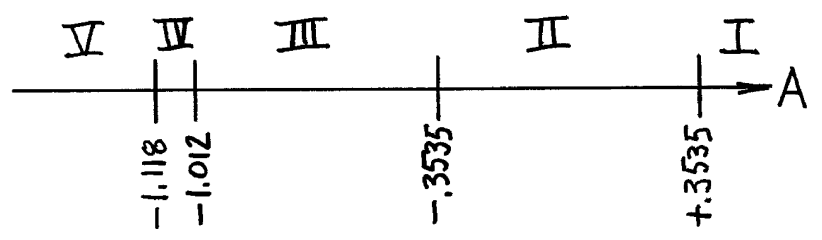

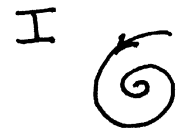
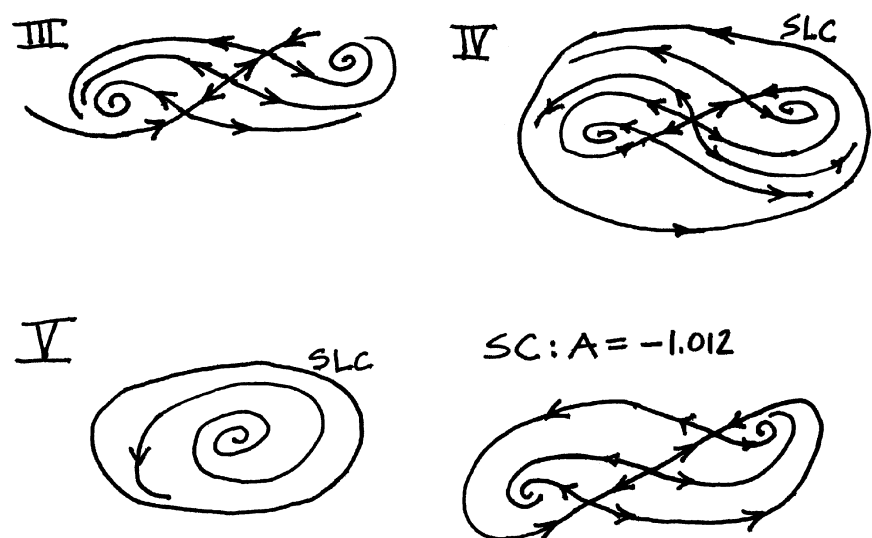

Figure 2. Sketch of slow flows in the $u-v$ plane for Example 2. ULC: unstable limit cycle, SLC: stable limit cycle. SC: saddleconnection bifurcation, which occurs at $A=-1.012$.

\section{Discussion}

The main difference between these two examples is that Example 1 involves the occurrence of a Hopf bifurcation, whereas Example 2 does not. Since both examples correspond to the parameter value $\Lambda=-1 / 2$, we may gain insight into the dependence of the dynamical structure on parameters by varying $\kappa$ and $A$ as in Figure 3. Here Example 1 corresponds to the horizontal line $\kappa=0$, and Example 2 corresponds to the horizontal line $\kappa=-1$. As can be seen from this figure, the branch of Hopf bifurcations exists for $\kappa>-3 / 4$. As expected, the horizontal line $\kappa=-3 / 4$, which corresponds to a system for which both the trace $(M)$ and the $\operatorname{det}(M)$ simultaneously vanish, lies between Examples 1 and 2 .

Although Figure 3 is drawn for the specific value $\Lambda=-1 / 2$, certain features of the bifurcation set will occur for a generic value of $\Lambda$. These include:

1. Places where the Hopf curve becomes tangent to the $P_{1}$ or $P_{4}$ saddle-node bifurcation curve and terminates. As just mentioned, both the trace $(M)$ and the $\operatorname{det}(M)$ vanish at such a point. The corresponding value of $\kappa$ is $\kappa=\frac{1}{2 \Lambda}-\frac{\Lambda}{2}$.

2. Places where the $P_{1}$ and $P_{2}$ bifurcation curves are tangent. By symmetry, the $P_{4}$ and $P_{3}$ curves are also tangent there. The associated value of $\kappa$ is $\kappa=-\Lambda$.

3. Places where the $P_{1}$ and $P_{4}$ branches go off to infinity. These come from the vanishing of the denominators in Equation (20) and correspond to $\kappa=\frac{1}{\Lambda}$. 


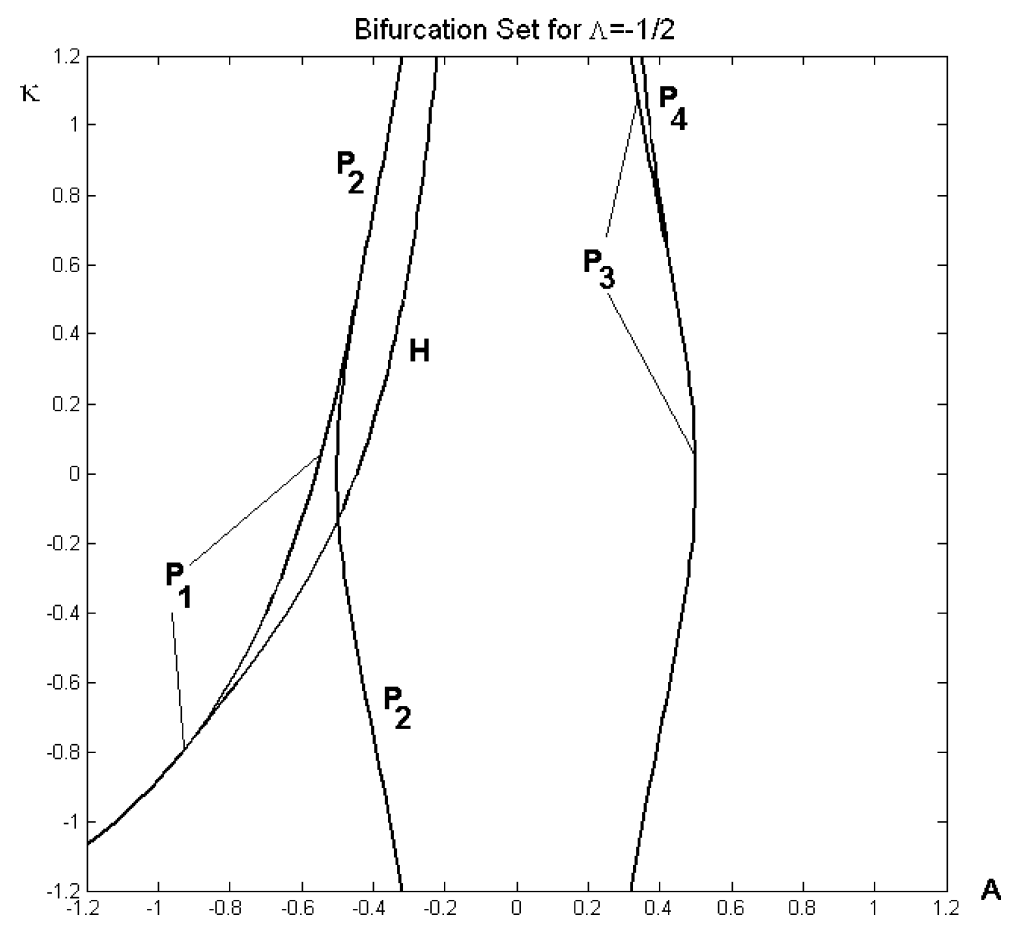

Figure 3. Partial bifurcation set in the $A-\kappa$ parameter plane for $\Lambda=-1 / 2, B=1, \beta_{1}=-6$ and $\beta_{2}=3 . P_{1}$ and $P_{4}$ are saddle-node bifurcations, $P_{2}$ and $P_{3}$ are pitchforks, and $H$ is Hopfs. These curves are given by Equations (20) and (27). Not shown are limit cycle folds, symmetry-breaking bifurcations and saddle-connection bifurcations (see Figures 1 and 2). We have no analytic expressions for these additional bifurcations.

\section{Conclusions}

We have studied the dynamics of a system (3) which exhibits the simultaneous phenomena of both parametric resonance and Hopf bifurcation. Imagine that we hold all parameters fixed except for the damping coefficient $A$, and that we ask what is expected to happen as $A$ decreases through the parametric resonance/Hopf region. We begin with a stable equilibrium point at the origin of the slow flow which corresponds to a stable trivial solution of Equation (3). Then the first bifurcation is reached while $A$ is still positive, giving rise to a pair of stable equilibria in the slow flow which correspond to a single stable period-2 subharmonic motion in Equation (3). As $A$ decreases and becomes negative, a variety of bifurcations may occur which culminate for sufficiently negative $A$ in a slow flow which exhibits only a stable limit cycle and an unstable equilibrium at the origin. This limit cycle corresponds in Equation (3) to a stable quasiperiodic motion which may be thought of as combining the periodic motion which would have been created in the Hopf (in the absence of parametric excitation) with a motion coming from the periodic parametric forcing.

In many applications, a limit cycle created in a Hopf bifurcation is destroyed in a saddle-connection bifurcation in the unforced system. This scenario occurs, for example, in the Takens-Bogdanov (doublezero eigenvalue) system (see [5], p. 371). Such a situation also occurs in the ENSO system [2,3] described briefly in the introduction. When such a system is parametrically excited, the analysis in the present paper shows that a quasiperiodic motion may be expected to occur in the neighborhood of the Hopf bifurcation. It is still an open question as to what happens to that motion as the bifurcation parameter proceeds towards the value corresponding to the saddle-connection bifurcation in the unforced system. 


\section{References}

1. Rand, R. H., Lecture Notes on Nonlinear Vibrations (Version 45), Published on-line by The Internet-First University Press, Ithaca, New York, 2004, http://dspace.library.cornell.edu/handle/1813/79.

2. Wang, B. and Fang, Z., 'Chaotic oscillations of tropical climate: A dynamic system theory for ENSO', Journal of Atmospheric Sciences 53, 1996, 2786-2802.

3. Wang, B., Barcilon, A., and Fang, Z., 'Stochastic dynamics of El Nino-Southern Oscillation', Journal of Atmospheric Sciences 56, 1999, 5-23.

4. Zalalutdinov, M., Aubin, K. L., Pandey, M., Zehnder, A. T., Rand, R. H., Craighead, H. T., and Parpia, J. M., 'Frequency entrainment for micromechanical oscillator', Applied Physics Letters 83, 2003, 3281-3283.

5. Guckenheimer, J. and Holmes, P., Nonlinear Oscillations, Dynamical Systems, and Bifurcations of Vector Fields, SpringerVerlag, New York, 1983.

6. Nayfeh, A. H., Perturbation Methods, Wiley, New York, 1973. 